\title{
A Multiobjective Model for Locating Drop-off Boxes for Collecting Used Products
}

\author{
Ken-ichi Tanaka* \\ Department of Informatics, Graduate School of Informatics and Engineering, \\ The University of Electro-Communications, Tokyo, Japan \\ Hirokazu Kobayashi \\ NTT COMWARE Corporation, Tokyo, Japan \\ Kenji Yura \\ Department of Informatics, Graduate School of Informatics and Engineering, \\ The University of Electro-Communications, Tokyo, Japan
}

(Received: January 31, 2013 / Revised: July 31, 2013 / Accepted: November 14, 2013)

\begin{abstract}
This paper proposes a multiobjective model describing the trade-offs involved in selecting the locations of drop-off boxes for collecting used products and transporting these products to designated locations. We assume the following reverse flow of used products. Owners of used products (cellular phones, digital cameras, ink cartridges, etc.) take them to the nearest drop-off box when the distance is reasonably short. We also assume that owners living closer to drop-off boxes dispose of more used products than do owners living farther from drop-off boxes. Different types of used products are collected, with each type requiring its own drop-off box. A transportation destination for each product is specified. Three objectives are considered: maximizing the volume of used products collected at drop-off boxes; minimizing the cost of transporting collected products to designated locations; and minimizing the cost of allocating space for drop-off boxes. We formulate the above model as a multiobjective integer programming problem and generate the corresponding set of Pareto optimal solutions. We apply the model to an area using population data for Chofu City, Tokyo, Japan, and analyze the trade-offs between the objectives.
\end{abstract}

Keywords: Reverse Logistics, Facility Location Problem, Multiobjective Optimization, Drop-off Boxes

*Corresponding Author, E-mail: ken1tnk@se.uec.ac.jp

\section{INTRODUCTION}

In today's supply chains, increasing attention is paid to the reverse flow of products from consumers to producers in addition to the traditional forward flow of products. The reverse flow of products is known as reverse logistics. An overview of recent developments regarding models and methods in reverse logistics can be found in Dekker et al. (2004).

The collection of used products is often regarded as an important first phase of reverse logistics. There are various approaches to organizing efficient collection of used products, such as on-site collection, unmanned dropoff sites, and staffed and smart drop-off sites (Beullens et al., 2004). This paper considers the problem of selecting locations for drop-off boxes to which owners take their used products. We consider used products, such as cellular phones, digital cameras, and ink cartridges, which are light enough to be carried by a single person and hence are suitable for collection using drop-off boxes.

The locations of drop-off boxes have a strong impact on the volume of collected used products, the cost 
of allocating space for boxes, and the cost of transporting the collected products to designated locations, all of which are important factors in the efficient and effective design of reverse logistics networks. Generally, these objectives are conflicting, and we, therefore, propose a multiobjective model to describe the trade-offs involved in the selection of locations for drop-off boxes with respect to these objectives.

Design problems for reverse logistics networks have received growing attention in the last two decades. See Beullens et al. (2004) and the review article by Melo et al. (2009) for recent development. The topic continues to attract many researchers and new models continue to be proposed-for example, Aras and Aksen (2008) and Das and Chowdhury (2012).

Usually the first phase in the design of a reverse network is the collection of used products from product holders. As such, the location of collection centers is a crucial factor that affects the total volume collected and also the total cost of transporting used products from end users. We review some of the papers that deal with the locations of collection centers below.

Jayaraman et al. (2003) propose a discrete facility location problem in which an efficient strategy is determined for the reverse logistics operations of hazardous products. Products located at retail outlets are first sent to collection sites and then transshipped to refurbishing sites. The model seeks to find the optimal number and location of collection and refurbishing facilities together with the flow of the hazardous products.

Min et al. (2006) also deal explicitly with the location of collection points. A nonlinear mixed integer model is presented for determining the optimal number and locations of collection points as well as centralized return centers. They note that one of the ways to improve the efficiency of product returns is improving customer convenience. Accordingly, a sufficient number of collection points need to be established near customer locations in order to reduce the travel time required of product holders to return used products.

The two aforementioned papers make the strong assumption that the volume of products returned by end users is known. In reality, however, the volume of returned products is strongly dependent on the distance from the location of the product holder to the nearest collection center. Very few papers explicitly model this aspect in the design of reverse network problems.

Aras and Aksen (2008) proposed a facility location-allocation model for modeling the return process in which the distance of the nearest collection center from the residence of the product holder affects the product holders' willingness to return a product. This model determines the optimal number and locations of collection centers as well as the optimal incentives for companies to offer to product holders. The model focuses on two factors that affect the willingness of the product holders to travel to a collection center to drop off their used products: the amount of the financial incentive offered at the time of the drop-off and the distance of the nearest collection center from the residence of the product holder. The model assumes only one type of used product.

In many cases, a single decision maker is responsible for the collection of several different types of used products at product-specific drop-off boxes. The final (or next) destination of collected products may differ depending on the types of products collected. In such situations, the new problem arises of choosing between the case of having fewer locations host multiple types of drop-off boxes and the case of having single drop-off boxes located at many locations. The preferable choice depends on the cost of transporting collected products to a next destination and the cost of securing the space for hosting at least one drop-off box. The proposed model incorporates this structure and can, therefore, determine the optimal locations of drop-off boxes for each product type, which is the key contribution of this paper.

The remainder of this paper is organized as follows. We describe the general setup and the proposed model in Section 2. A mathematical formulation of the proposed problem is provided in Section 3. We apply our model to population data for Chofu City, Tokyo, Japan, and analyze the obtained Pareto optimal solutions in Section 4. Finally, in Section 5, we conclude the paper.

\section{MODEL DESCRIPTION}

In this section, we set up the problem and state our assumptions regarding the behavior of product owners.

\subsection{Problem Setup}

We focus on a geographical area in which two types of used products, denoted A and B, are distributed (Figure 1). This can easily be extended to the case where $n$ types of products exist, a formulation for which is given in the next section. We consider used products, such as cellular phones, digital cameras, and ink cartridges, which are all light enough to be carried by a single person. Drop-off boxes are product-specific; in other words, a drop-off box for product A cannot be used to collect product $B$ and vice versa.

Owners take used products to drop-off boxes, and the collected used products are then transported to designated facilities. The facilities may be disassembly centers where returned products are inspected, graded, and sorted, or may be remanufacturing facilities where product recovery is performed. Three objectives are considered: 1) maximizing the total volume of used products A and B collected at drop-off boxes; 2) minimizing the cost of transporting collected products to designated locations; and 3) minimizing the cost for allocating space for drop-off boxes. We develop a model describing the trade-offs involved in selecting the locations of drop-off boxes for collecting used products and transporting the collected products to designated locations. 


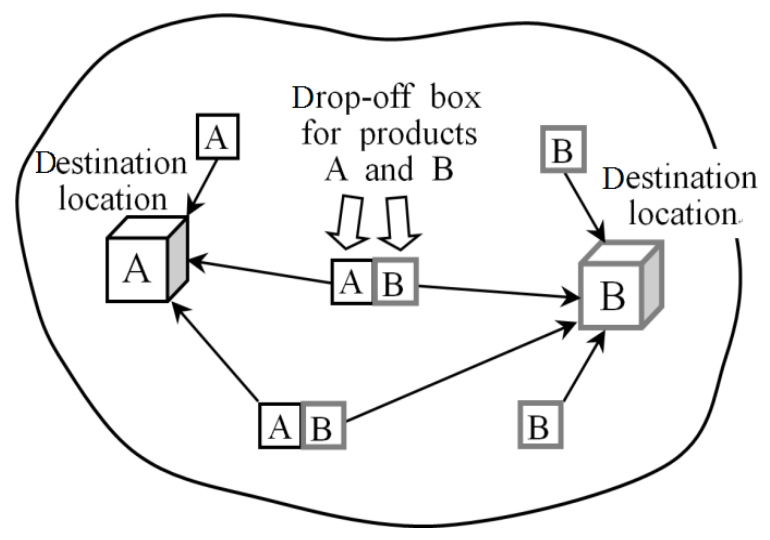

Figure 1. Reverse flow of used products.

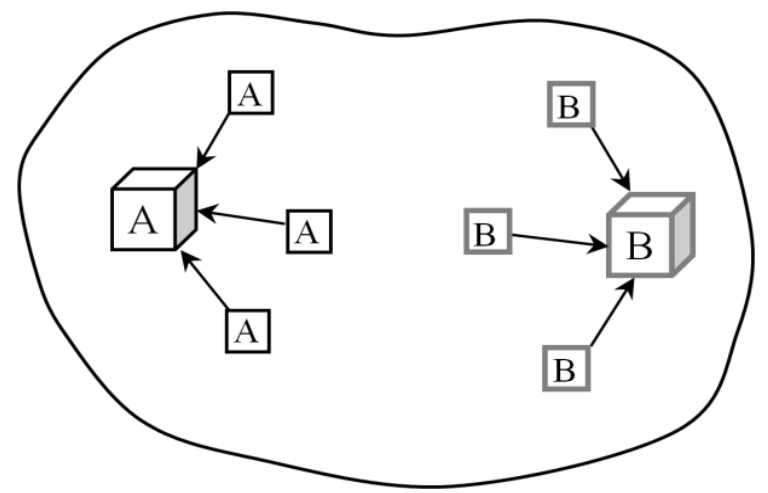

Figure 2. Typical preferable distribution of drop-off box locations when transportation cost is high.

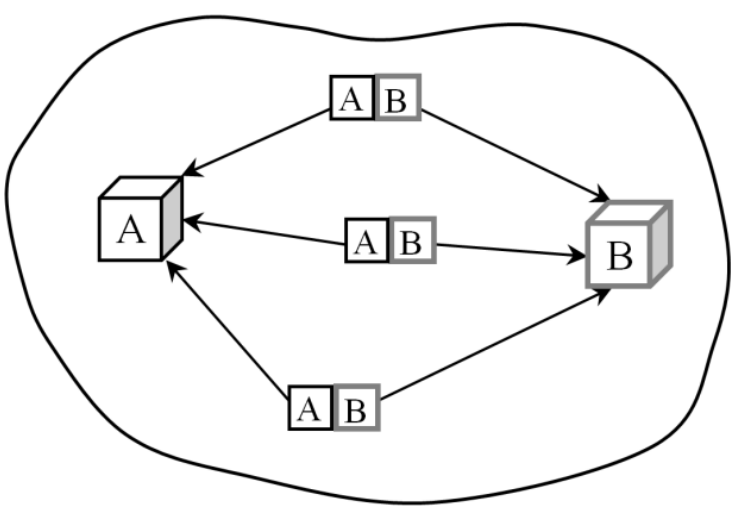

Figure 3. Typical preferable distribution of drop-off box locations when space allocation cost is high.

The preferable distribution of drop-off box locations strongly depends on the importance assigned to two types of costs: transportation cost and space allocation cost. When the weight assigned to the transportation cost is greater, drop-off boxes for each product tend to be sited closer to the corresponding designated destination (Figure 2). When the weight assigned to the space allocation cost is greater, drop-off boxes for products A and $\mathrm{B}$ tend to be co-located (Figure 3). The proposed model can describe both cases by assigning a suitable weight to each objective.

\subsection{Assumptions}

We make the following assumptions about the behavior of product owners.

Assumption 1. Owners of used products use the nearest drop-off boxes.

Assumption 2. The proportion of product owners who take used products to drop-off boxes is a decreasing function of the distance to the nearest drop-off boxes.

Two scenarios can be considered based on the purpose of the trip taken by owners. In one scenario, the purpose of the trip is specifically to take used products to their respective drop-off boxes, while in the other, owners of used products stop at drop-off boxes along a preplanned route, such as the daily commute to work. We assume the former scenario, where Assumption 1 is quite natural. To describe this behavior of product owners, constraints necessary to assign owners to their respective nearest boxes are introduced into the problem formulation.

In general, the distance from the location of a product owner to the nearest drop-off box strongly affects the behavior of the owner, with more used products expected to be collected from owners living closer to dropoff boxes than from those living farther from drop-off boxes. Assumption 2 is introduced to describe this structure. This type of modeling approach can be found in the literature on facility location, where the value of a service can be described as a function of the distance to the nearest facility - for example, Church and Roberts (1983). In the numerical example presented here, we assume that the volume of used products collected per person decreases exponentially with distance.

\section{FORMULATION}

This section presents a formulation of the proposed problem where $n$ types of products exist. First, some notation is introduced, followed by an integer programming formulation of the problem. The planning model assumes a unit period, for example, six months or two years.

\subsection{Notation}

The following sets and parameters are used in the problem formulation:

\section{Sets}

I : set of demand locations, indexed by $i$, for drop-off boxes for all types of used products.

$J$ : set of candidate locations, indexed by $j$ for drop-off boxes for all types of used products. 
$P$ : set of all types of used products: $P=\{1,2, \cdots, n\}$, indexed by $p$

\section{Parameters}

$l^{p}:$ number of drop-off boxes for product $p \in P$.

$c^{p}:$ cost of transporting a unit volume of product $p \in P$ per unit distance.

$q_{i}^{p}$ : total volume of used product $p \in P$ generated at demand location $i \in I$ per unit period.

$s_{j}$ : space allocation cost at candidate location $j \in J$ per unit period.

$d_{j}^{p}$ : distance between candidate location $j \in J$ and destination for product $p \in P$.

$v_{i j}^{p}$ : volume of used product $p \in P$ collected at $j \in J$ from demand location $i \in I$ per unit period.

$\alpha_{p}$ : minimum required proportion of collected volume for product type $p \in P$ to that of the total volume collected for all product types.

The first objective of the proposed model is maximizing the sum of the total collected volumes for all product types per unit period. Under this objective, the collected volume of one product type may be greatly less than that of another type. To prevent this, we introduce $\alpha_{p}$ which takes a value between 0 and 1 , and assume that at least $100 \alpha_{p} \%$ of the volume of used products of type $p \in P$ is collected compared with the total volume collected.

We introduce an additional notation. Let $[\mathrm{m}]_{i}$ be the index of the $m$-th closest candidate location from demand location $i \in I$. This notation is used in describing the closest assignment constraints.

We introduce the following binary variables:

$z_{j}$ : binary variable that takes a value of 1 when at least one drop-off box is located at candidate location $j \in J$ and 0 otherwise.

$x_{j}^{p}$ : binary variable that takes a value of 1 when a dropoff box for product $p \in P$ is located at candidate location $j \in J$ and 0 otherwise.

$y_{i j}^{p}$ : binary variable that takes a value of 1 when owners of used product $p \in P$ living at $i \in I$ are assigned to a drop-off box for product $p$ at candidate location $j \in J$ and 0 otherwise.

\subsection{Integer Programming Formulation}

With the above notation, the proposed problem can be formulated as follows:

$$
\begin{array}{ll}
\operatorname{maximize} & \sum_{p \in P} \sum_{i \in I} \sum_{j \in J} v_{i j}^{p} y_{i j}^{p} \\
\operatorname{minimize} & \sum_{p \in P} \sum_{i \in I} \sum_{j \in J} c^{p} d_{j}^{p} v_{i j}^{p} y_{i j}^{p} \\
\operatorname{minimize} & \sum_{j \in J} s_{j} z_{j} \\
\text { subject to } & \sum_{j \in J} x_{j}^{p}=l^{p} \quad \forall p \in P
\end{array}
$$

$$
\begin{gathered}
x_{j}^{p} \leq z_{j} \quad \forall j \in J, \forall p \in P \\
y_{i j}^{p} \leq x_{j}^{p} \quad \forall i \in I, \forall j \in J, \forall p \in P \\
\sum_{j \in J} y_{i j}^{p}=1 \quad \forall i \in I, \forall p \in P \\
x_{[m]_{i}}^{p} \leq \sum_{k=1}^{m} y_{i,[]_{i}}^{p} \quad \forall i \in I, \forall p \in P, \\
m=1,2, \cdots,|J|-1 \\
\sum_{i \in I} \sum_{j \in J} v_{i j}^{p} y_{i j}^{p} \geq \alpha_{p} \sum_{p \in P} \sum_{i \in I} \sum_{j \in J} v_{i j}^{p} y_{i j}^{p}, \\
\quad \forall p \in P \\
z_{j} \in\{0,1\} \quad \forall j \in J \\
x_{j}^{p} \in\{0,1\} \quad \forall j \in J, \forall p \in P \\
y_{i j}^{p} \in\{0,1\} \quad \forall i \in I, \forall j \in J, \forall p \in P
\end{gathered}
$$

Objective (1) represents maximization of the sum of the total collected volumes for all product types per unit period. Objective (2) is the total cost of transporting used products for all types collected at all drop-off boxes per unit period. The first and second objectives are conflicting in nature. Larger volumes of used products collected at drop-off boxes contribute more to the first objective, while at the same time they increase the total transportation cost and thus negatively affect the second objective. Objective (3) is the total space allocation cost per unit period. This cost incorporates factors such as expenses for personnel to maintain the boxes and to collect used products at places with at least one drop-off box.

Constraint (4) limits the number of boxes to be located for product type $p \in P$ to $l^{p}$. Constraint (5) permits locating drop-off boxes for product type $p \in P$ at only candidate locations where space is allocated for them. Constraint (6) restricts the assignment of demand locations $i \in I$ to only candidate locations $j \in J$ where drop-off boxes are located for product type $p \in P$.

Constraints (7) and (8) represent Assumption 1, according to which owners of used products for each type use the nearest drop-off boxes. Under constraint (7), each demand location is assigned to exactly one candidate location for each product type. Constraint (8) is required to account for the tendency of owners to visit the nearest drop-off boxes. For each product type, these constraints stipulate that if a drop-off box is located at the $m$-th closest candidate location to demand location $i$, this demand has to be assigned to one of the candidate locations from among the first to the $m$-th closest from the demand. The closest assignment constraint used in this paper is presented in detail in Daskin (1995). Further information on this topic can be found in Gerrard and Church (1996).

Constraint (9) does not allow imbalance in the amounts of collected products for all product types. Finally, constraints (10) through (12) are standard binary constraints on decision variables. 


\section{NUMERICAL EXAMPLES}

In this section, we present some numerical examples applied to population data for Chofu City in Tokyo. First, the problem instances are explained, after which the Pareto optimal solutions obtained for these instances are analyzed.

\subsection{Problem Instance}

Figure 4 shows the problem instance, which uses population data for Chofu City. Black disks represent 105 regions, where the area of each disk is proportional to the population of the corresponding region. The total population is 216,119 as of the 2005 Census. We consider the case in which two product types, A and B, exist. Both $q_{i}^{\mathrm{A}}$ and $q_{i}^{\mathrm{B}}$ are set equal to the total population. In other words, each person has one used product $A$ and one used product B. The destination locations for products $\mathrm{A}$ and $\mathrm{B}$ are indicated by black and gray diamonds, respectively. There are 25 candidate locations for dropoff boxes for products A and B, as denoted by black and gray squares.

Regarding the proportion of product owners visiting the nearest drop-off box, we employ a function which decreases exponentially with increasing Euclidean distance to the box. The proportion is assumed to decrease to 1/e at $500 \mathrm{~m}$ away from the drop-off box compared with that at $0 \mathrm{~m}$ (Figure 5). Using this function, $v_{i j}^{\mathrm{A}}$ and $v_{i j}^{\mathrm{B}}$ are given as follows:

$$
\begin{aligned}
& v_{i j}^{\mathrm{A}}=q_{i}^{\mathrm{A}} \exp \left(-d_{i j} / 500\right), \\
& v_{i j}^{\mathrm{B}}=q_{i}^{\mathrm{B}} \exp \left(-d_{i j} / 500\right),
\end{aligned}
$$

While the Euclidean distance is different from the shortest path distance over a road network, various studies indicate that the Euclidean distances is a good measure for approximating distances on real road networks

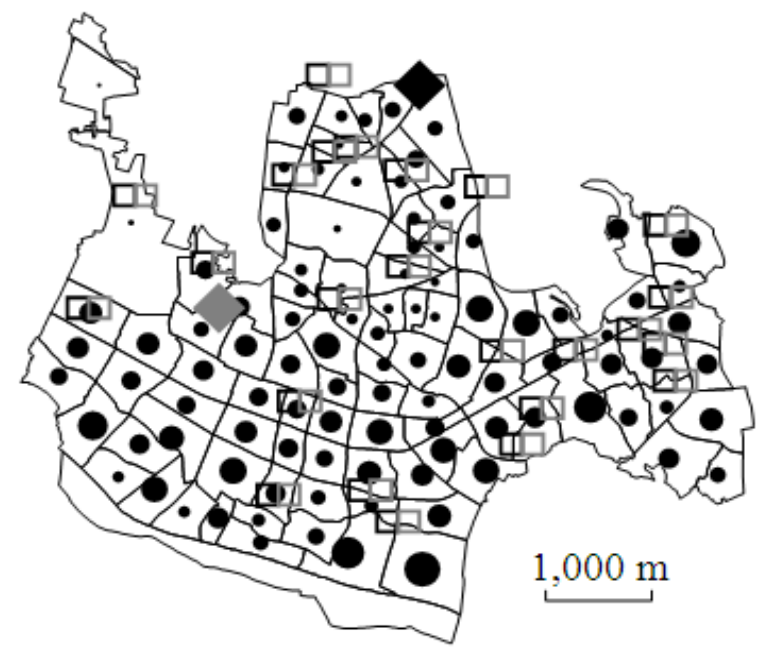

Figure 4. Problem instance: Chofu City.

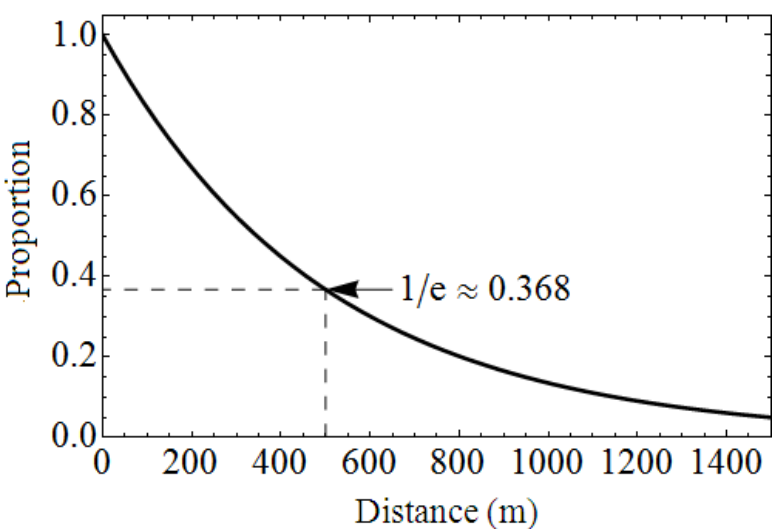

Figure 5. Proportion of product owners visiting the nearest drop-off box as the distance increases.

by appropriately multiplying a scaling factor (e.g., 1.25). See Koshizuka and Kobayashi (1983) and Love and Morris (1979) for a discussion of this. We set the cost of transporting a unit volume of products $\mathrm{A}$ and $\mathrm{B}$ to be $c^{\mathrm{A}}=c^{\mathrm{B}}=0.005$. We also assume that at least $40 \%$ of the used products of one type are collected relative to the total volume of both types collected, and therefore $\alpha^{\mathrm{A}}=\alpha^{\mathrm{B}}=0.4$. The following two problem instances are considered:

Instance 1. $p^{\mathrm{A}}=p^{\mathrm{B}}=3, s_{j}=1,000 \quad \forall j \in J$

Instance 2. $p^{\mathrm{A}}=p^{\mathrm{B}}=3, s_{j}=10,000 \quad \forall j \in J$

Instance 2 places greater importance on space allocation cost than Instance 1. Thus, Instance 1 assumes a scenario similar to the one shown in Figure 2, and Instance 2 is illustrated in Figure 3.

\subsection{Pareto Optimal Solutions}

We consider a bi-objective version of the proposed problem. The first objective is given by Eq. (1). For the second objective, we combine the two types of costs given by the sum of Eqs. (2) and (3). Pareto optimal solutions are enumerated by the constraint method (Cohon, 1978) for Instances 1 and 2 and calculated by using IBM ILOG CPLEX 12.4.

Figure 6 shows the trade-off between collected volume and total cost for Instances 1 and 2 . There are 155 Pareto optimal solutions for Instance 1 and 91 for Instance 2. The leftmost solutions, indicated by 1-a and 2-a for each instance, are the ones in which the total cost is minimized, while the rightmost solutions, indicated by $1-\mathrm{d}$ and 2-d, are the ones where the collected volume is maximized. There are various compromise solutions between these two extreme solutions.

First, let us focus on four Pareto optimal solutions (1-a, 1-b, 1-c, and 1-d) for Instance 1, which are shown in Figure 7. In the case of the cost-minimizing solution 1-a, all three drop-off boxes for products $\mathrm{A}$ and $\mathrm{B}$ are co-located, and their locations are close to both destina- 
tion locations, resulting in a drastic reduction in total cost. On the other hand, in the volume-maximizing solution 1-d, the distribution of the locations of drop-off boxes is quite different from that in 1-a. In solution 1-d, boxes are more spread out and located in areas around which large volumes of used products are expected to be collected. At the same time, however, the boxes are located without paying any attention to the designated destinations, which results in high transportation cost. Compromise solutions 1-b and 1-c combine both characteristics, where drop-off boxes for products A and B are co-located at only one site in 1-b and two sites in 1-c. Solution 1-c can be regarded as a better candidate than solution 1-d since the total cost is more than $26 \%$ lower than that in 1-d, whereas the decrease in collected volume is only about $8 \%$.

Next, we focus on four Pareto optimal solutions (2a, 2-b, 2-c, and 2-d) for Instance 2. Only two of these (2$\mathrm{b}$ and 2-c) are shown in Figure 8 since solutions 2-a and 2-d are exactly the same as 1-a and 1-d, respectively. In contrast to solutions 1-b and 1-c, all three pairs of dropoff boxes are co-located in solutions 2-b and 2-c. This is due to the higher space allocation cost. As in the case of 1-c and 1-d, solution 2-c can be regarded as a better candidate than solution 2-d since the total cost is more than $20 \%$ lower than that in 1-d, whereas the decrease in collected volume is only about $8 \%$.

We also obtained Pareto optimal solutions for $p^{\mathrm{A}}=p^{\mathrm{B}}=4$ and $p^{\mathrm{A}}=p^{\mathrm{B}}=5$ for the two different space allocation costs. The solutions obtained for these cases have similar characteristics to $p^{\mathrm{A}}=p^{\mathrm{B}}=3$. Table 1 lists the average number of locations where at least one dropoff box is located. It also shows the total number of Pareto optimal solutions in brackets. It can be seen that drop-off boxes tend to be co-located when the space allocation cost is higher, as intuitively expected. The number of Pareto optimal solutions increases proportionally to the number of drop-off boxes and tends to be larger at lower space allocation cost. This is caused by the larger variability in solutions in the latter case due to the distribution with both individually located and colocated boxes.

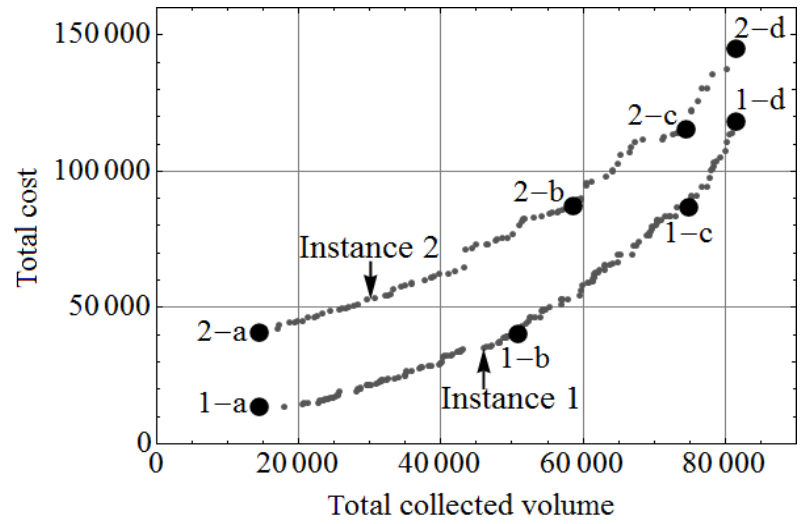

Figure 6. Trade-off between total collected volume and total cost.
Table 1. Average number of locations where at least one drop-off box is located and total number of Pareto optimal solutions

\begin{tabular}{ccc}
\hline & $s_{j}=1,000$ & $s_{j}=10,000$ \\
& $\forall j \in J$ & $\forall j \in J$ \\
\hline$p^{\mathrm{A}}=p^{\mathrm{B}}=3$ & $5.12(155)$ & $3.22(91)$ \\
$p^{\mathrm{A}}=p^{\mathrm{B}}=4$ & $6.32(253)$ & $4.19(144)$ \\
$p^{\mathrm{A}}=p^{\mathrm{B}}=5$ & $7.46(351)$ & $5.13(185)$ \\
\hline
\end{tabular}
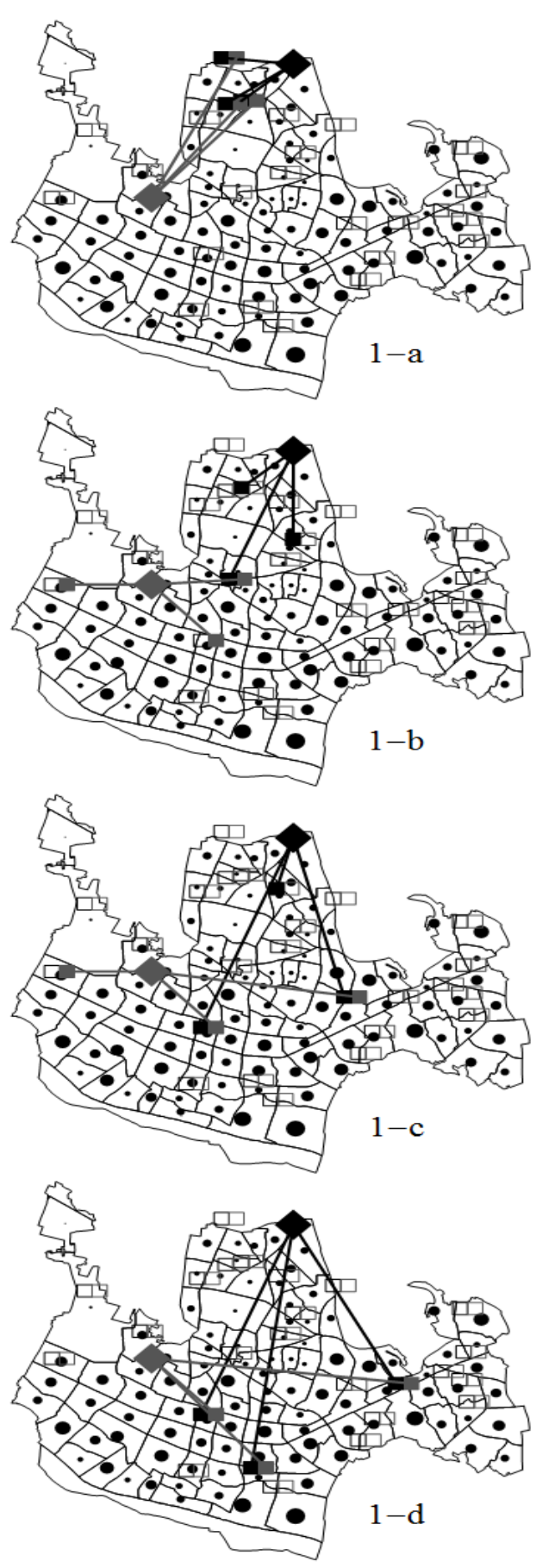

Figure 7. Four Pareto optimal solutions for Instance 1. 


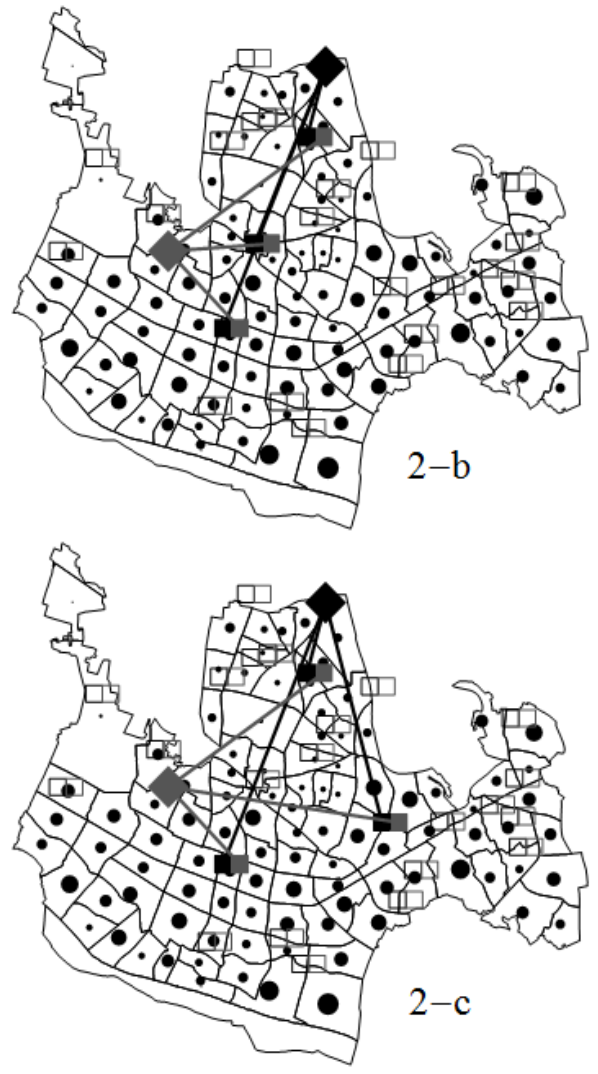

Figure 8. Two Pareto optimal solutions for Instance 2.

There are real world instances to which the proposed model can be applied. One such example is a recycling initiative called the "Ink Cartridge Homecoming Project" that was established by six printer manufacturers (Brother Industries Ltd., Canon, Dell Co. Ltd., Seiko Epson Corporation, Hewlett-Packard Japan Ltd., and Rex mark International Inc.).

The purpose of this project is to promote collection and recycling of used ink-cartridges by locating drop-off boxes at municipal offices or post offices throughout Japan. In the first phase of this recycling network, owners of used products bring their cartridges to a drop-off box. In designing this type of recycling network, the locations of drop-off boxes will have a strong impact on the total volume collected and also the cost associated with the collection and transportation. The proposed problem can serve as a basic model for designing this type of real world problem.

\section{CONCLUSION}

We proposed a model for selecting locations for drop-off boxes for collecting used products whose weight is low enough to allow them to be carried, such as cellular phones, digital cameras, and ink cartridges. We focused on the volume of collected products, the cost of transporting them to designated locations, and the cost of space allocation for the drop-off boxes, all of which are important elements in designing efficient and effective reverse logistics networks. We developed a multiobjective model to describe the trade-offs involved in the selection of locations for drop-off boxes for these objectives.

We assumed that a higher volume of used products is expected from owners located closer to drop-off boxes than from owners located farther from them. Several types of used products were considered, each requiring its own type of drop-off box, and a transport destination for each product was also specified.

An integer programming formulation of the proposed problem was presented. The model was applied to actual population data for Chofu City, and generated a set of Pareto optimal solutions. Some of the obtained solutions performed well in terms of the collected volume while realizing much lower transportation and space allocation costs than solutions maximizing the volume.

There are various directions for extending the proposed problem. One important aspect is to model more accurately the product holders' willingness to return used products. This paper considered only the proximity to the collection center measured by the travel distance to the nearest drop-off box as having an effect on the return rate. Aras and Aksen (2008) introduced the amount of financial incentive offered at the time of drop-off and assume that this affects the return rate. Incorporation of this factor into the proposed framework would be an interesting topic to consider in the future.

\section{ACKNOWLEDGMENTS}

The author would like to thank the two anonymous referees for their valuable comments and suggestions, which greatly improved the first version of the paper.

\section{REFERENCES}

Aras, N. and Aksen, D. (2008), Locating collection centers for distance- and incentive-dependent returns, International Journal of Production Economics, 111 (2), 316-333.

Beullens, P., Van Oudheusden, D., and Van Wassenhove, L. N. (2004), Collection and vehicle routing issues in reverse logistics, In: Reverse Logistics, Springer, Heidelberg, Germany, 95-134.

Church, R. L. and Roberts, K. L. (1983), Generalized coverage models and public facility location, $\mathrm{Pa}$ pers of the Regional Science Association, 53(1), 117-134.

Cohon, J. L. (1978), Multiobjective Programming and Planning, Academic Press, New York, NY.

Das, K. and Chowdhury, A. H. (2012), Designing a reverse logistics network for optimal collection, re- 
covery and quality-based product-mix planning, $I n-$ ternational Journal of Production Economics, 135 (1), 209-221.

Daskin, M. S. (1995), Network and Discrete Location: Models, Algorithms, and Applications, Wiley, New York, NY.

Dekker, R., Fleischmann, M., Inderfurth, K., and Van Wassenhove, L. N. (2004), Reverse Logistics: Quantitative Models for Closed-Loop Supply Chain, Springer, Heidelberg, Germany.

Gerrard, R. A. and Church, R. L. (1996), Closest assignment constraints and location models: properties and structure, Location Science, 4(4), 251-270.

Jayaraman, V., Patterson, R. A., and Rolland, E. (2003), The design of reverse distribution networks: models and solution procedures, European Journal of
Operational Research, 150(1), 128-149.

Koshizuka, T. and Kobayashi, J. (1983), The road distances and straight line distances, Proceedings of the Annual Conference of the City Planning Institute of Japan, 18, 43-48.

Love, R. F. and Morris, J. G. (1979), Mathematical models of road travel distances, Management Science, 25(2), 130-139.

Melo, M. T., Nickel, S., and Saldanha-da-Gama, F. (2009), Facility location and supply chain management: a review, European Journal of Operational Research, 196(2), 401-412.

Min, H., Ko, H. J., and Ko, C. S. (2006), A genetic algorithm approach to developing the multi-echelon reverse logistics network for product returns, Omega, 34(1), 56-69. 